\title{
In the consideration of internal friction forces in nonstationary dynamics problems
}

\author{
Yuriy E. Senitsky ${ }^{1}$, and Elena N. Elekina ${ }^{1 *}$ \\ ${ }^{1}$ Samara State Technical University, Institute of Architecture and Civil Engineering, \\ 194, Molodogvardeyskaya St., 443001, Samara, Russia
}

\begin{abstract}
In this paper, the original methodology for taking into consideration internal friction forces is given in the example of nonstationary oscillations of a beam with elastically clamped edges. Bearing in mind the experimentally confirmed fact that the forces of internal friction practically do not affect the forms of structural vibrations, they are introduced into the equation of motion after separation of the spatial variable. This decomposition approach of forming a mathematical model in conjunction with the frequency independent Voigt hypothesis, with a known loss factor, made it possible to represent the solution in the form of spectral decomposition. For this purpose, we used the structural algorithm of the finite integral transform (FIT) method with the definition of the transformation kernel in the solution process. In fact, the proposed method is a method of quasinormal coordinates and represents an effective method of solving dynamic problems for mechanical systems in the presence of internal friction forces.
\end{abstract}

\section{Introduction}

Spectral expansions obtained as a result of the application of the method of integral transformations with finite limits (FIT) represent the effective solution of linear nonstationary problems of mechanics. One of the main conditions that determines the possibility of such an approach is the self-adjointness of the initial-boundary value problem under consideration. At the same time, as a result of taking into consideration the resistance forces, including internal friction, this important property of the mathematical model is lost, and a direct way of constructing an analytical solution is impossible [1-8]. However, the structural algorithm of FIT method developed by the author [9-10] in combination with the frequency independent Voigt hypothesis [11] by introducing viscous drag forces in the solution process made it possible to obtain a new qualitative solution of the corresponding dynamic problems. In this paper, this approach is demonstrated by the example of a nonstationary problem for a beam under sufficiently general conditions for its fixing and loading.

* Corresponding author: Elekina-E1@yandex.ru 


\section{Materials and methods}

We consider an elastic beam (prismatic rod $\mathrm{EJ}=$ const) loaded with a transverse dynamic load $\mathrm{q}(\mathrm{x}, \mathrm{t})$ distributed along the span $\mathrm{x} \in(\mathrm{o}, \mathrm{l})$ and in time $\mathrm{t}>0$ in an arbitrary law. A rather general case of elastic pinching of the end sections of a rod with a known coefficient of elasticity $\alpha$ is investigated. The material of a beam is homogeneous, isotropic. The mathematical model takes into account the forces of internal friction, with a given loss factor for each mode of oscillation $\gamma_{-} \mathrm{i}(\mathrm{i}=(1, \infty))$. The solution algorithm proposed below is based on the method of quasinormal coordinates [11] and corresponds to an experimentally confirmed fact about the significant effect of internal friction forces on the behavior of structures in time and practically does not affect the shape of the oscillations. For this purpose, the structural algorithm of the method of finite integral transformations (FIT) is very convenient, in the procedure of which all the components of the resulting solution are determined. It is essential that the results obtained in this case reflect the nature of the damped oscillation process.

Differential equations of motion and boundary conditions in the study of transverse oscillations of a beam are formulated as follows [9]:

$$
\begin{gathered}
\text { EJ } \frac{\partial^{4} y(x, t)}{\partial x^{4}}+m \frac{\partial^{2} y(x, t)}{\partial t^{2}}=q(x, t), \\
\left.y(x, 0)\right|_{t=0}=y_{0}(x),\left.\quad \frac{\partial y(x, t)}{\partial t}\right|_{t=0}=v_{0}(x), \\
\left.y(x, t)\right|_{x=0,1}=0, \quad \text { EJ } \frac{\partial^{2} y(x, t)}{\partial x^{2}}= \pm\left.\alpha \frac{\partial y(x, t)}{\partial x}\right|_{x=0,1},
\end{gathered}
$$

Here $\mathrm{y}(\mathrm{x}, \mathrm{t})$ is the required function of transverse displacements, $\mathrm{J}$ and $\mathrm{m}$ are respectively the moment of inertia and the distributed mass of the beam, y_ $0, v_{-} 0$ are the initial displacements and the velocity of displacements. Relations (2) and (3) represent the initial and boundary conditions for the boundary value problem under consideration. It should be noted that the forces of internal friction are introduced later in the process of solving the problem (1) - (3).

We introduce a finite integral transformation (FIT) with respect to the variable $\mathrm{x}$, defined by such a pair of formulas [9]:

$$
\begin{aligned}
& \bar{y}\left(\lambda_{i}, t\right)=\int_{0}^{1} y(x, t) G\left(\lambda_{i}, x\right) d x ; \\
& y(x, t)=\sum_{i=1}^{s} \bar{y}\left(\lambda_{i}, t\right) G\left(\lambda_{i}, x\right)\left\|G_{i}\right\|^{-2} .
\end{aligned}
$$

Where $y^{-}\left(\lambda \_i, t\right)$ is the transform of the original y $(x, t), G\left(\lambda \_i, x\right)$ is the kernel of the integral transformations defined below, $\lambda \_i$ are the positive parameters $i=((1, \infty) \overline{)}$.

$$
\left\|G_{\mathrm{i}}\right\|^{2}=\int_{0}^{1} \mathrm{G}^{2}\left(\mathrm{O}_{\mathrm{i}}, \mathrm{x}\right) \mathrm{dx}
$$

- square of the norm of a function $\left(\lambda_{i}, x\right)$

Relations (4) are valid in the case when the functions $G\left(\lambda_{-} i, x\right)$ represent an orthogonal system.

We apply FIT (4) to the equation (1) and the initial conditions (2). We have

$$
\mathrm{EJ} \int_{0}^{1} \frac{\partial^{4} \mathrm{y}}{\partial \mathrm{x}^{4}} \mathrm{Gdx}+\mathrm{m} \frac{\partial^{2}}{\partial \mathrm{t}^{2}} \int_{0}^{1} \mathrm{yGdx}=\int_{0}^{1} \mathrm{qGdx}
$$




$$
\left.\int_{0}^{1} \mathrm{yGdx}\right|_{\mathrm{t}=0}=\int_{0}^{1} \mathrm{y}_{0} \mathrm{Gdx},\left.\quad \frac{\partial}{\partial \mathrm{t}} \int_{0}^{1} \mathrm{yGdx}\right|_{\mathrm{t}=0}=\int_{0}^{1} \mathrm{~V}_{0} \mathrm{Gdx} .
$$

Here, in accordance with (4), the quadratures in equalities (6), (7) represent the transformants of the functions $q(x, t), y \_0(x), V \_0(x)$, i.e.

$$
\left\{\overline{\mathrm{q}}\left(\lambda_{\mathrm{i}}, \mathrm{t}\right), \overline{\mathrm{y}_{0}}\left(\lambda_{\mathrm{i}}\right), \overline{\mathrm{V}_{0}}\left(\lambda_{\mathrm{i}}\right)\right\}=\int_{0}^{1}\left\{\mathrm{q}(\mathrm{x}, \mathrm{t}), \mathrm{y}_{0}(\mathrm{x}), \mathrm{V}_{0}(\mathrm{x})\right\} \mathrm{G}\left(\lambda_{\mathrm{i}}, \mathrm{x}\right) \mathrm{dx} \text {. }
$$

Using the algorithmic procedure of the structural algorithm of FIT method [1-4], we integrate the first quadrature (6) by parts. As a result, we obtain

$$
\begin{aligned}
& \operatorname{EJ}\left\{\left.\Phi(y, G)\right|_{0} ^{1}+\int_{0}^{1} y G^{I V} d x\right\}+m \frac{\partial^{2} g\left(\lambda_{i}, t\right)}{\partial t^{2}}=\bar{q}\left(\lambda_{i}, t\right), \\
& \left.\Phi(\mathrm{y}, \mathrm{G})\right|_{0} ^{1}=\left.\left[\frac{\partial^{3} \mathrm{y}}{\partial \mathrm{x}^{3}} \mathrm{G}-\frac{\partial^{2} \mathrm{y}}{\partial \mathrm{x}^{2}} \mathrm{G}^{\prime}+\frac{\partial \mathrm{y}}{\partial \mathrm{x}} \mathrm{G}^{\prime \prime}-\mathrm{y} G^{\prime \prime \prime}\right]\right|_{0} ^{1} \text {. } \\
& \text { 1) }\left.\Phi(y, G)\right|_{0} ^{1}=0, \quad \text { 2) } \int_{0}^{1} \mathrm{yG}^{\mathrm{V}} \mathrm{dx}=\lambda_{\mathrm{i}}^{4} \int_{0}^{1} \mathrm{yGdx} \text {. }
\end{aligned}
$$

Taking into account (8), (10), (11), equations (9) and conditions (7) take the form:

$$
\begin{gathered}
\frac{\partial^{2} \mathrm{y}\left(\lambda_{\mathrm{i}}, \mathrm{t}\right)}{\partial \mathrm{t}^{2}}+\omega_{\mathrm{i}}^{2} \overline{\mathrm{y}}\left(\lambda_{\mathrm{i}}, \mathrm{t}\right)=\frac{1}{\mathrm{~m}} \overline{\mathrm{q}}\left(\lambda_{\mathrm{i}}, \mathrm{t}\right) ; \\
\left.\left.\overline{\mathrm{y}}\left(\lambda_{\mathrm{i}}, 0\right)\right|_{\mathrm{t}=0}=\overline{\mathrm{y}_{0}}\left(\lambda_{\mathrm{i}}\right) ; \quad \frac{\mathrm{d}}{\mathrm{dt}} \overline{\mathrm{y}} \lambda_{\mathrm{i}}, \mathrm{t}\right)\left.\right|_{\mathrm{t}=0}=\overline{\mathrm{v}}_{0}\left(\lambda_{\mathrm{i}}\right)
\end{gathered}
$$

where $\omega_{\mathrm{i}}=\lambda_{\mathrm{i}}^{2} \sqrt{\frac{\mathrm{EI}}{\mathrm{m}}}$ circular oscillation frequencies of the beam.

Relations (11) allow us to formulate a differential equation and boundary (boundary) conditions for the kernel of the integral transformation $G\left(\lambda \_i, x\right)$. We have

$$
\begin{gathered}
G^{\mathrm{IV}}\left(\lambda_{\mathrm{i}}, \mathrm{x}\right)-\lambda_{\mathrm{i}}{ }^{4} \mathrm{G}\left(\lambda_{\mathrm{i}}, \mathrm{x}\right)=0 ; \\
\left.\mathrm{G}\left(\lambda_{\mathrm{i}}, \mathrm{x}\right)\right|_{\mathrm{z}=0,1}=0,\left.\quad \mathrm{G}^{\prime \prime}\left(\lambda_{\mathrm{i}}, \mathrm{x}\right) \mp \mu \mathrm{G}^{\prime}\left(\lambda_{\mathrm{i}}, \mathrm{x}\right)\right|_{\mathrm{x}=0,1}=0, \quad \mu=\alpha(\mathrm{E})^{-1}
\end{gathered}
$$

\section{Results}

Thus, as a result of the application of the QIB (4), the original boundary value problem (1) (3) has split into two - (12), (13) and (14), (15), i.e. there was a separation of variables. In accordance with the method of quasinormal coordinates and the frequency-independent Voigt hypothesis, we introduce into the differential equation (12) the forces of internal friction at each mode of oscillation $\gamma_{i} \omega_{i} \frac{d y\left(\lambda_{i}, t\right)}{d t}$. We have

$$
\frac{\partial^{2} \mathrm{y}\left(\lambda_{\mathrm{i}}, \mathrm{t}\right)}{\partial \mathrm{t}^{2}}+\gamma_{\mathrm{i}} \omega_{\mathrm{i}} \frac{\mathrm{dy}\left(\lambda_{\mathrm{i}}, \mathrm{t}\right)}{\mathrm{dt}}+\omega_{\mathrm{i}}^{2} \overline{\mathrm{y}}\left(\lambda_{\mathrm{i}}, \mathrm{t}\right)=\mathrm{m}^{-1} \overline{\mathrm{q}}\left(\lambda_{\mathrm{i}}, \mathrm{t}\right)
$$

The solutions of equations (16) and (14), taking into account conditions (13), are written in the form:

$$
\begin{gathered}
\bar{y}\left(\lambda_{\mathrm{i}}, \mathrm{t}\right)=\mathrm{e}^{-\beta_{\mathrm{i}} \mathrm{t}}\left[\bar{y}_{0}\left(\lambda_{\mathrm{i}}\right) \cos \alpha_{\mathrm{i}} \mathrm{t}+\left(\beta_{\mathrm{i}} \overline{\mathrm{y}}_{0}\left(\lambda_{\mathrm{i}}\right)+\overline{\mathrm{v}}_{0}\left(\lambda_{\mathrm{i}}\right) \alpha_{\mathrm{i}}{ }^{-1} \sin \alpha_{\mathrm{i}} \mathrm{t}\right]+\right. \\
+\left(\alpha_{\mathrm{i}} \mathrm{m}\right)^{-1} \int_{0}^{\mathrm{t}} \overline{\mathrm{q}}\left(\lambda_{\mathrm{i}}, \tau\right) \mathrm{e}^{-\beta_{\mathrm{i}}(\mathrm{t}-\tau)} \sin \alpha_{\mathrm{i}}(\mathrm{t}-\tau) \mathrm{d} \tau ; \\
\mathrm{G}\left(\lambda_{\mathrm{i}}, \mathrm{x}\right)=\mathrm{C}_{1 \mathrm{i}} \operatorname{ch} \lambda_{\mathrm{i}} \mathrm{x}+\mathrm{C}_{2 \mathrm{i}} \operatorname{sh} \lambda_{\mathrm{i}} \mathrm{x}+\mathrm{C}_{3 \mathrm{i}} \cos \lambda_{\mathrm{i}} \mathrm{x}+\mathrm{C}_{4 \mathrm{i}} \sin \lambda_{\mathrm{i}} \mathrm{x}
\end{gathered}
$$




$$
\alpha_{i}=\sqrt{\omega_{i}^{2}-\left(0,5 \gamma_{i} \omega_{i}\right)}, \quad \beta_{i}=0.5 \gamma_{i} \omega_{i}
$$

For various dynamic effects $\mathrm{q}(\mathrm{x}, \mathrm{t})$ on the beam, in expression (17) it is only necessary to calculate the Duhamel integral.

The arbitrary constants $C_{-} 1 \mathrm{i}, \mathrm{C} \_2 \mathrm{i}, \mathrm{C} \_3 \mathrm{i}, \mathrm{C} \_4 \mathrm{i}$ are determined as a result of substitution of the relation (20) into the boundary conditions (16). In this case C_3i $=-\mathrm{C}_{-} 1 \mathrm{i}$, and then a non-trivial solution of the resulting system of the remaining three algebraic equations is sought. Equating its principal determinant to zero, we find

$$
\left|\begin{array}{ccc}
2 \lambda_{\mathrm{i}} & -\mu & -\mu \\
\left(\operatorname{ch} \lambda_{\mathrm{i}} 1-\cos \lambda_{\mathrm{i}} 1\right) & \operatorname{sh} \lambda_{\mathrm{i}} \mathrm{l} & \sin \lambda_{\mathrm{i}} 1 \\
\left.\lambda_{\mathrm{i}}\left(\operatorname{ch} \lambda_{\mathrm{i}} 1+\cos \lambda_{\mathrm{i}} 1\right)\right]+\mu\left(\operatorname{sh} \lambda_{\mathrm{i}} 1+\sin \lambda_{\mathrm{i}} 1\right) & \left(\lambda_{\mathrm{i}} \operatorname{sh} \lambda_{\mathrm{i}} 1+\mu \operatorname{ch} \lambda_{\mathrm{i}} \mathrm{l}\right) & \left(\mu \cos \lambda_{\mathrm{i}} \mathrm{l}-\lambda_{\mathrm{i}} \sin \lambda_{\mathrm{i}} \mathrm{l}\right)
\end{array}\right|=0_{\mathrm{i}}
$$

The roots of the transcendental equation (20) are the parameters $\lambda \_i$ and, in accordance with (14), the oscillation frequencies $\omega$ i can be determined. Substituting expressions (17) into the inversion formula (4), and taking into account the C_1i, C_2i, C_3i, C_4i and (18) found, we obtain the required displacements of the beam $y(x, t)$.

\section{Discussion}

Using the method of quasinormal coordinates, the structural algorithm for the finite integral transforms, and the frequency independent Voigt hypothesis, a new analytic solution of the dynamical problem for the rod is constructed in the presence of internal friction forces. The results are obtained for arbitrary conditions of loading and fixing the rod. Spectral decompositions are constructed by decomposition of a mathematical model, and all components of the reduced structure (load integral, integral transformation core, oscillation frequencies) are determined directly in the process of solving.

\section{Conclusions}

In conclusion, it should be noted that the numerical analysis of the calculation of the frequencies and modes of oscillations of an anisotropic cylinder and a sphere in the presence of internal friction forces is given in the author's papers. The proposed methodology of consideration of viscous drag forces can also be useful in studying the oscillations of the bottoms of navigable locks.

\section{References}

1. D.A. Shlyakhin, Procedia Engineering, 150, pp. 68-73 (2016)

2. D.A. Shlyakhin, O.V. Kazakova, Procedia Engineering 153, 662-666 (2014)

3. V.S. Shirokov, I.S. Kholopov, A.V. Solovejv, Procedia Engineering 153, 655-661 (2016)

4. A.A. Abashkin, Russian Math. (Iz. VUZ) 60:2, 1-6 (2016)

5. A.O. Lukin, V.Y.Alpatov, D.D. Chernyshev, Urban Construction and Architecture 2, 4 - 9 (2016) DOI 10.17673/Vestnik.2016.02.1

6. A.A.Romanov, O.V. Leonov, S.V. Evdokimov, Urban Construction and Architecture 4, 66-70 (2011) DOI 10.17673/Vestnik.2011.04.13 
7. K.V. Vorob'eva, K.P. Zainulabidova, M.V. Frize, Vestnik of Dagestan Satate technical University. Technical Sciences 1(40), 108 - 119 (2016)

8. A.M. Uzdin, D.V. Schelkunov, Seismic resistant construction. Safety of structures 2, 47-50 (2011)

9. Y.E. Senitsky, Vestnik Samara State Technical University. Series «Physics and mathematics» 2(19), 78 - 89 (2009)

10. Y.E. Senitsky, Procedia Engineering 153, 649 - 654 (2016)

11. A.I. Tseitlin, A.A. Kusainov, Methods of accounting for internal friction in dynamic design calculations (1987) 